\title{
RIGHT SIDED SIGMOID COLON AND REDUNDANT DESCENDING COLON ON CONVENTIONAL AND CT IMAGING
}

\author{
Mandeep Singh', Madhan Kumar², Daisy Gupta ${ }^{3}$
}

1 Junior Resident, Department of Radiodiagnosis, Government Medical College, Amritsar, Punjab, India.

2Junior Resident, Department of Radiodiagnosis, Government Medical College, Amritsar, Punjab, India.

${ }^{3}$ Assistant Professor, Department of Radiodiagnosis, Government Medical College, Amritsar, Punjab, India.

HOW TO CITE THIS ARTICLE: Singh M, Kumar M, Gupta D. Right sided sigmoid colon and redundant descending colon on conventional and CT imaging. J. Evolution Med. Dent. Sci. 2018;7(44):5617-5620, DOI: 10.14260/jemds/2018/1073

\section{CASE PRESENTATION}

A 62-year-old male presented with history of severe constipation, abdominal distension, haemorrhoids and blood in stool in surgical OPD of Guru Nanak Dev Hospital, Amritsar. The patient was referred for barium studies of colon, which showed a loop of colon in pelvic region (at normal location of ileal loops) and redundant and long descending colon extending across midline to reach hepatic flexure on right and continuing as sigmoid colon on right side. Transverse colon and ascending colon were normal in length and position. On CECT abdomen of the patient, a long segment of descending colon was identified. Its first part stretched obliquely from the splenic flexure to the right side traversing the umbilical quadrant, then it turned right of midline at the level of L5 vertebra. Later it turned upward and toward the right, ascending up to the level of body of L2 vertebra. The third part descended obliquely on the medial side of the ascending colon up to the pelvic brim. The fourth part was in the lesser pelvis and continued as the sigmoid colon in the right iliac fossa. The inferior mesenteric artery was seen arising from right side of ventral surface of abdominal aorta opposite third lumbar vertebra.

Descending colon is part of large intestine which lies along the left side of abdomen, posterior to left kidney. It ends in sigmoid colon, which is situated in pelvis and ends in rectum at S3 level. While descending colon is a retroperitoneal structure, sigmoid colon is suspended by mesocolon. Various case reports are reported in literature regarding displaced descending colon discovered during anatomic dissection. Very few case reports have been made regarding identification of redundant colon in a clinical patient. We present a case of redundant and right sided descending colon with right sided sigmoid colon and double hepatic flexure, which has long redundant segments on barium enema and CECT abdomen. There is also associated variations in blood vessels supplying these anomalous colons. Case was confirmed on CECT abdomen.

'Financial or Other Competing Interest': None.

Submission 03-09-2018, Peer Review 11-10-2018,

Acceptance 17-10-2018, Published 29-10-2018.

Corresponding Author:

Dr. Daisy Gupta,

Assistant Professor,

Department of Radiodiagnosis,

Government Medical College,

Amritsar, Punjab, India.

E-mail: daisygupta23@gmail.com

DOI: $10.14260 /$ jemds/2018/1073

\section{Investigations}

\section{On Plain X-Ray Abdomen}

No abnormal air-fluid levels were seen. There were no abnormal radio-opaque shadows seen. Bilateral psoas shadows and soft tissue shadows were identified as normal.

\section{On Barium Enema}

After filling the rectum, the contrast was identified as filling the sigmoid colon, which was present anomalously towards the right side. Filling of barium outlined the extension of colon from sigmoid on right side with coiling in right iliac fossa and hypogastrium. This loop of colon extended towards right hepatic flexure and then turned downwards towards umbilicus. It was seen turning left to form splenic flexure and then continue as transverse colon, ascending colon and caecum in right iliac fossa. Provisional diagnosis of redundant descending colon was considered, and patient was advised CT evaluation for confirmation, Figure 1 and Figure 2.

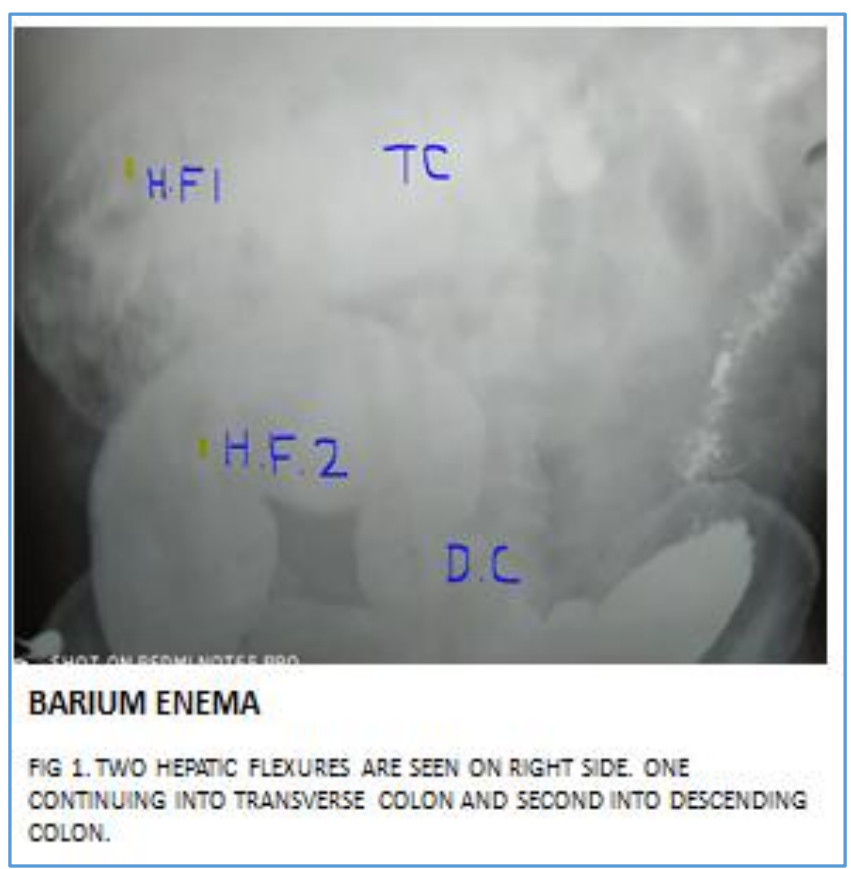

Figure 1. Shows Two Hepatic Flexures Figure 2. Shows the Course of Descending Colon and Sigmoid Colon 


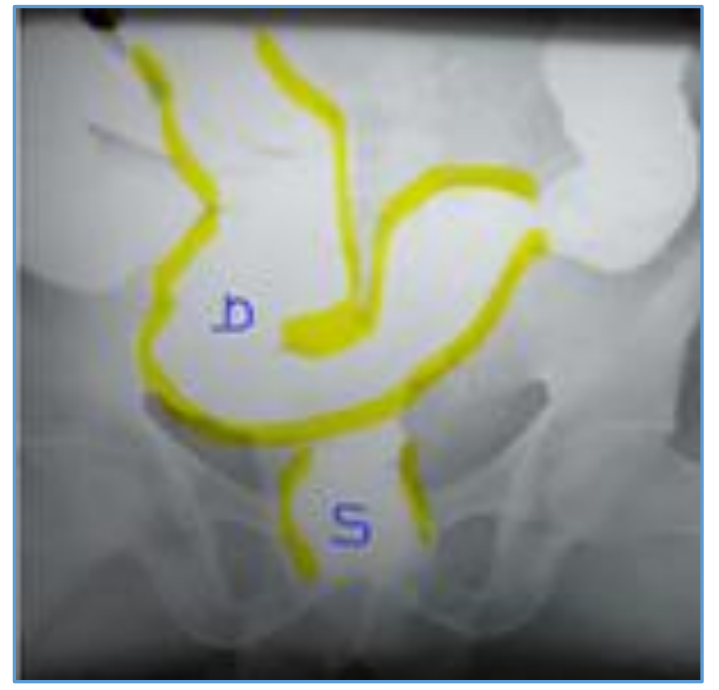

Descending colon can be clearly seen turning right. Then upwards. Its descent as sigmoid colon posterior to it can also be identified.

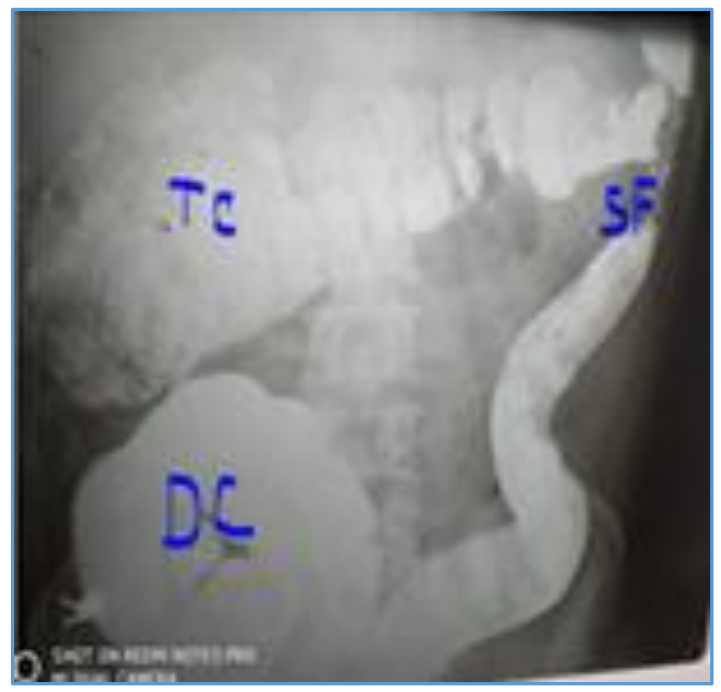

Coiled descending colon can be seen to right it is seen crossing to left and continuing as transverse colon

\section{On CECT Abdomen with Oral and Per Rectal Contrast}

Both kidneys, liver and spleen were normal in their position and orientation of Stomach, duodenum, jejunum and ileum were identified normally filled with oral contrast. Duodenum formed its normal C-loop. Caecum and ascending colon were present on right side as retroperitoneal structure, ascended up to the liver, turned left at hepatic flexure to continue as transverse colon till splenic flexure. A long redundant segment of descending colon was identified. The first part stretched obliquely from the splenic flexure to the right side, traversing the umbilical quadrant, then at the right of the midline at the level of L5 vertebrae it turned upward and toward the right, ascending up to the level of body of L2 vertebrae. The third part descended obliquely on the medial side of the ascending limb up to the pelvic brim. After coiling, the fourth part in the lesser pelvis continued as the sigmoid colon in the right iliac fossa. The inferior mesenteric artery was seen arising from right side of ventral surface of abdominal aorta, opposite third lumbar vertebra, Figure 5.6.7.
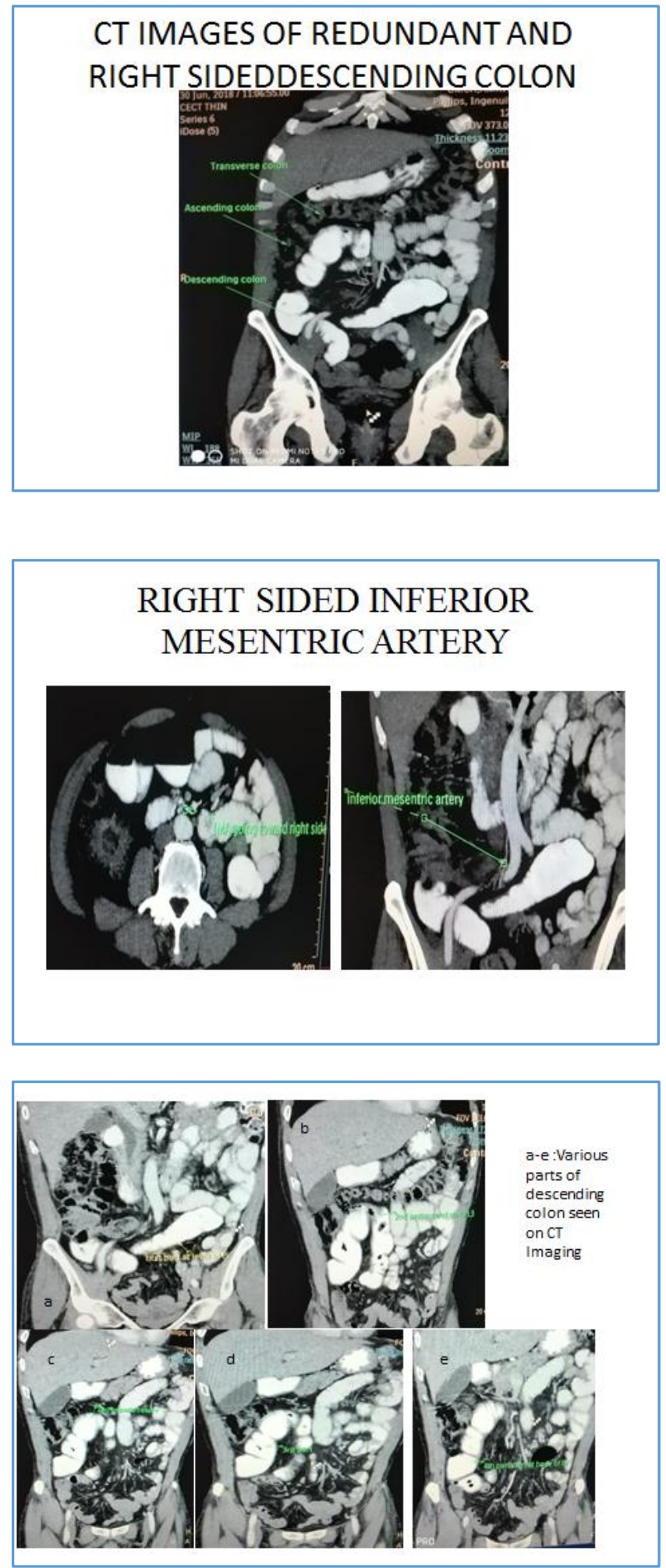

a-e. Various parts of descending colon seen on CT imaging

In this case the observations simulate the type II variety of the redundant colon to some extent and type IV variety, because here the elongated loop of descending colon formed the second hepatic flexure and there was also right-sided sigmoid colon. ${ }^{1}$ 


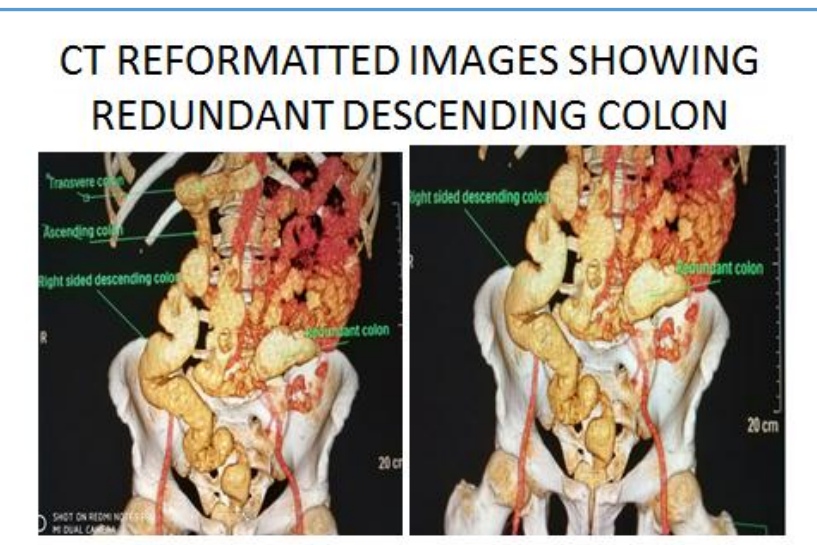

According to the classification described by Kanagasuntheram et al, ${ }^{1}$ our case had double hepatic flexure (Group II) and sigmoid colon is on right side (Group IV).

\section{DISCUSSION OF MANAGEMENT}

Patient was treated conservatively with symptomatic treatment after the diagnosis was explained to him.

The embryonic gut develops from three parts: Foregut, midgut and the hindgut. The large intestine develops mainly from the hindgut loop. It extends from the distal end of ileum at ileocecal junction in right iliac fossa to the anus (About 1.5 metres in length). It includes caecum, appendix, ascending colon, transverse colon (Extending from hepatic flexure to splenic flexure), descending colon, sigmoid colon, rectum and anal canal. Ascending and descending colon are retroperitoneal structures. Phrenicocolic ligament is attached to the splenic flexure. The retroperitoneal descending colon measures about $25 \mathrm{~cm}$ long and descends on left side through the left hypochondrium and lumbar regions. In left iliac fossa it curves medially and downwards to continue as the sigmoid colon. The descending colon and sigmoid colon are supplied by the inferior mesenteric artery. The sigmoid colon (pelvic colon) measures about $40 \mathrm{~cm}$ in length. It first descends along the left pelvic wall and turns postero-inferior towards S3 to continue as rectum. Here, it lies between rectum and the urinary bladder.

Both the descending colon and sigmoid colon are known to possess redundant loops. Descending and sigmoid colons develop from the hindgut. In the early stage of development, both of them possess their mesocolons. However, later on during development, the descending colon loses its mesocolon to become retroperitoneal. During embryonic period, failure of disappearance of descending mesocolon may lead to persistence of descending mesocolon in postnatal life and the length of descending colon is variable and depends on the segmental obliteration of dorsal mesentery. If a short segment of mesocolon is fused, then only a small part of descending colon is retroperitoneal and rest of it becomes extremely long and mobile resulting in redundant colon. ${ }^{2}$ Sigmoid colon also might vary in length, may be right-sided and may form redundant loops. Though the anomalous positions of the sigmoid colon are well-known, the variations in the position of the descending colon are very rare.

In Kantor's ${ }^{3}$ definition the redundant colon is one which is too long to fit into the body of its owner without undergoing reduplication. According to Kantor's any case in which the enema-filled pelvic loop rises above a line joining the iliac crests is diagnosed redundant colon. ${ }^{4}$ Redundant colon refers to the extra bends in the colon by Kanagasuntheram et al, who classified redundant colon into four groups 1 :

Group I: Presence of complete ascending and descending mesocolon,

Group II: Presence of double hepatic flexure.

Group III: An extension of sigmoid colon into the abdominal cavity.

Group IV: Displacement of sigmoid colon towards the right side.

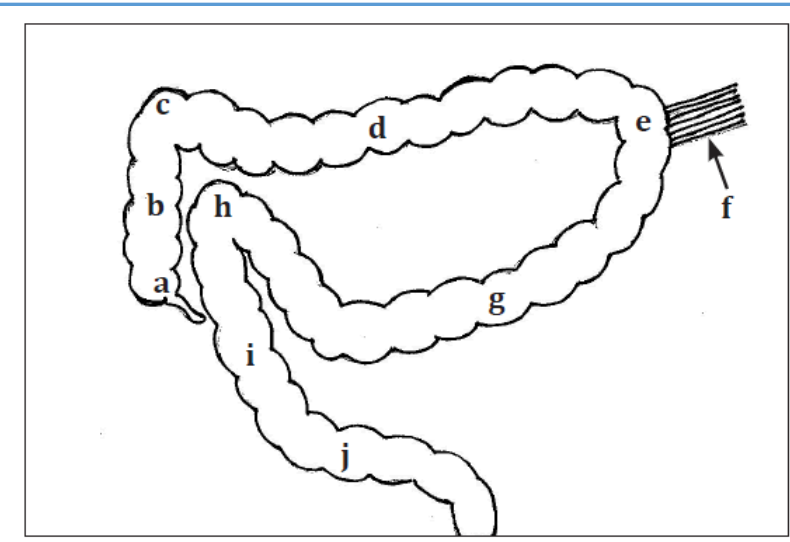

Figure 1. The illustration shows the variations of colon. (a: caecum and appendix; $\boldsymbol{b}$ : ascending colon; $\boldsymbol{c}$ : first hepatic flexure; $\boldsymbol{d}$ : transverse colon; $e$ : splenic flexure; $f$ : phrenico-colic ligament; $g$ : redundant descending colon; $\boldsymbol{h}$ : second hepatic flexure; $\boldsymbol{i}$ : right sided descending colon; $\boldsymbol{j}$ : right sided sigmoid colon)

Variations are most commonly seen in the sigmoid colon in terms of length and position, whereas literature shows very few cases of variations in the position and length of the descending colon. Displacement of the descending colon to the right, specifically to the midline is an extremely rare variation.

Numerous cases regarding the variant colon have been reported. However, only a few have been reported regarding the variations of descending colon specifically. Bhadoria et al described a case with vertical segment extending to L4-5 and horizontal part crossing to right across various vessels, nerves, ureter etc. Inferior mesenteric artery arose from right side of aorta. ${ }^{5}$ Jacob et al also described a case of short and high ascending colon with redundant descending colon on right side. ${ }^{6}$ Nayak et al described a case of coiled descending colon and straight sigmoid colon. ${ }^{7}$ Firef et al described a case where descending colon was lying between the left kidney and left psoas major. ${ }^{8}$ Developmental anomalies of descending colon in association with renal anomalies has been documented by Balthazar. ${ }^{2}$ Few cases have been described in literature of variable length and position of descending colon during dissection in cadavers in adult Indian males. Srivastava et al reported a right sided descending colon. ${ }^{9}$ A displaced mesocolon along with sigmoid colon has been reported by Nayak et al, 10 in which the descending colon descended in the midline of the abdomen. A redundant loop of descending colon that formed a second hepatic flexure has been reported by Gupta et al.11 Clinical case reports reporting anomalies in colon have been reviewed by Balthazar. ${ }^{2}$ A case of knot formation in transverse and sigmoid colon due to twisting of a hyperactive bowel segment over a passive segment of bowel was reported 
by McBrearty et al, which lead to lower abdominal pain and constipation. ${ }^{12}$ Pyrtek et al studied a case on barium meal with right sided sigmoid colon. ${ }^{13}$ Komiyama et al reported a case of a 50-year-old Japanese male with excessively long distal part of the colon including the right-sided sigmoid colon. ${ }^{14}$

Variations in the position and length of the descending and sigmoid colons are significant for radiologists and surgeons. Variant case can lead to confusion during barium enema and CT scan. Surgeons may find it difficult during colonoscopy and sigmoidoscopy and may cause inadvertent perforation of colon and other complications if they are not aware of the possibility of variations. Brummer et al studied 106 patients radiologically with complaints of constipation and 53 patients of enema without constipation and found redundant colon in $46 \%$ of patients with constipation more than 10 years. While the incidence of redundant colon was $15 \%$ in cases of constipation less than 10 years. ${ }^{4}$

The descending colon when loaded with faeces can compress the aorta, leading to altered blood supply to the lower part of the body. It may cause compression of bladder resulting in frequent micturition. ${ }^{10}$ Volvulus of colon can occur when there is axial twisting of the colon on its vascular pedicle. Sharma et al has described a case of malrotation with transverse colon volvulus in a patient presenting with early pregnancy and acute intestinal obstruction. ${ }^{15}$ Other presentations are acute or chronic constipation, indefinite discomfort over the colon, indigestion, loss of weight, insomnia and pain are some of the symptomatology patients present with

\section{Learning Points}

1. Congenital anomalies of gut should always be kept in mind while performing radiographic studies.

2. This case illustrates long redundant and right sided descending colon, which has travelled to the right of abdomen to form a second hepatic flexure associated with right sided sigmoid colon.

3. Modern imaging modalities and algorithms like maximum intensity projection have enabled the diagnosis as well as confirmation of congenital variants of gut, especially colon.

4. Timely radiographic diagnosis will also guide surgeons in their managements and avoid complications during colonoscopy and sigmoidoscopy like perforation, obstruction of view and unnecessary interventions.

\section{CONCLUSION}

Anomalies in the position of colon can go undetected throughout life. But modern diagnostic technologies have made it possible to diagnose these anomalies and thus assist the surgeons and gynaecologists. The right sided position of the descending colon is extremely rare, and it might get compressed by the jejunum and ileum or it might compress the aorta which is situated behind it.

Descending colon is part of large intestine which lies along the left side of abdomen, posterior to left kidney. It ends in sigmoid colon, which is situated in pelvis and ends in rectum at S3 level. While descending colon is a retroperitoneal structure, sigmoid colon is suspended by mesocolon. Various case reports are reported in literature regarding displaced descending colon discovered during anatomic dissection. Very few case reports have been made regarding identification of redundant or right sided descending colon in a clinical patient. We present a case of redundant right sided descending colon with right sided sigmoid colon and double hepatic flexure seen on barium enema and CECT abdomen. There is also associated variations in blood vessels supplying these anomalous colons. Case was confirmed on CT abdomen.

\section{REFERENCES}

[1] Kanagasuntheram R, Kin LS. Observations on some anomalies of the colon. Singapore Medical Journal 1970;11(2):110-7.

[2] Balthazar EJ. Congenital positional anomalies of the colon: radiographic diagnosis and clinical implications. II. Abnormalities of fixation. Gastrointestinal Radiology 1977;2(1):49-56.

[3] Kantor JL. Anomalies of the colon: their roentgen diagnosis and clinical significance. Résumé of ten years' study. Radiology 1934;23(6):651-62.

[4] Brummer P, Seppala P, Wegelius U. Redundant colon as a cause of constipation. Gut 1962;3(2):140-1.

[5] Bhadoria P, Bakshi S, Agarwal S, et al. Right sided sigmoid colon and redundant loop of descending colon with its embryological correlation and clinical significance. Journal of Clinical and Diagnostic Research 2016;10(3):AD05-AD07.

[6] Jacob M, Shivarama CH, Bindu S, et al. Undescended caecum and appendix with right sided sigmoid colon a case report. Nitte University Journal of Health Sciences 2013;(3)4:125-7.

[7] Nayak SB, Swamy RS, Aithal AP, et al. Coiled descending colon with persistent mesocolon and a straight sigmoid colon - an unique congenital anomaly. Online $\mathrm{J}$ of Health Allied Sciences 2016;15(2):8.

URL:

http://www.ojhas.org/issue58/2016-2-8.html

[8] Eiref SD, Holekamp S, Winestone M, et al. Descending colon interposition in a patient presenting with abdominal pain and acute appendicitis. International Journal of Anatomical Variations 2010;3:146-8.

[9] Shrivastava P, Tuli A, Kaur S, et al. Right sided descending and sigmoid colon: its embryological basis and clinical implications. Anatomy \& Cell Biology 2013;46(4):299-302.

[10] Nayak SB, Pamidi N, Shetty SD, et al. Displaced sigmoid and descending colons: a case report. OA Case Reports 2013;2(17):166.

[11] Gupta I, Majumdar S, Mandal S. A redundant loop of descending colon and right sided sigmoid colon. International Journal of Anatomical Variations 2012;5:11-3.

[12] McBrearty A, Harris A, Gidwani A. Transverse-sigmoid colon knot: a rare cause of bowel obstruction. Ulster Medical Journal 2011;80(2):107-8.

[13] Pyrtek LJ, Jenney WL. Fixed retrocolic right sided dolichosigmoid colon. Ann Surg 1960;151(2):268-73.

[14] Komiyama M, Shimada Y. A case of a right-sided sigmoid colon. Kaibogaku Zasshi 1991;66(6):537-40.

[15] Sharma D, Parameshwaran R, Dani T, et al. Malrotation with transverse colon volvulus in early pregnancy: a rare cause for acute intestinal obstruction. British Medical Journal Case Reports 2013. doi:10.1136/bcr2013-200820. 